\title{
PARAMETER ESTIMATION FOR MACROSCOPIC PEDESTRIAN DYNAMICS MODELS FROM MICROSCOPIC DATA*
}

\author{
SUSANA N. GOMES ${ }^{\dagger}$, ANDREW M. STUART ${ }^{\ddagger}$, AND MARIE-THERESE WOLFRAM ${ }^{\S}$
}

\begin{abstract}
In this paper we develop a framework for parameter estimation in macroscopic pedestrian models using individual trajectories - microscopic data. We consider a unidirectional flow of pedestrians in a corridor and assume that the velocity decreases with the average density according to the fundamental diagram. Our model is formed from a coupling between a density dependent stochastic differential equation and a nonlinear partial differential equation for the density, and is hence of McKean-Vlasov type. We discuss identifiability of the parameters appearing in the fundamental diagram from trajectories of individuals, and we introduce optimization and Bayesian methods to perform the identification. We analyze the performance of the developed methodologies in various situations, such as for different in- and outflow conditions, for varying numbers of individual trajectories, and for differing channel geometries.
\end{abstract}

Key words. macroscopic pedestrian models, generalized McKean-Vlasov equations, parameter estimation, optimization-based and Bayesian inversion

AMS subject classifications. 35K20, 35R30, 62F15

DOI. $10.1137 / 18 \mathrm{M} 1215980$

1. Introduction. The complex dynamics of large pedestrian crowds as well as constant urbanization has initiated many developments in the field of transportation research, physics, urban planning, and, more recently, applied mathematics. Originally, model development was mostly based on empirical observations. More recently, a plethora of data, from video surveillance cameras as well as experiments, is starting to become available. This data, usually individual trajectories, is used to calibrate individual-based models or to identify characteristic relations, such as the fundamental diagram, which have the potential to be observed in large pedestrian crowds. In this paper we study such a calibration approach, setting up parameter estimation methodologies which allow us to estimate parameters in macroscopic pedestrian models using individual trajectories.

There is a rich literature on mathematical models for pedestrian dynamics; see [12]. Individual-based models, also known as microscopic models, are among the most popular in the engineering and transportation literature. In these models individuals are characterized by their position and sometimes velocity; see, for example, [23]. On the macroscopic level the crowd is described by a density. These models are often derived from first principles, since the rigorous transition from the micro- to

\footnotetext{
* Received by the editors September 21, 2018; accepted for publication (in revised form) May 23, 2019; published electronically August 6, 2019.

https://doi.org/10.1137/18M1215980

Funding: The work of the first and second authors was supported by the EPSRC Programme grant EQUIP. The work of the first author was also supported by the Leverhulme Trust via the Early Career Fellowship ECF-2018-056. The work of the second and third authors was supported by a Royal Society international collaboration grant. The work of the third author was partially supported by the Austrian Academy of Sciences via the New Frontier's grant NST-001.

${ }^{\dagger}$ Mathematics Institute, University of Warwick, Coventry CV4 7AL, UK (susana.gomes@warwick. ac.uk).

${ }^{\ddagger}$ Department of Computing and Mathematical Sciences, Caltech, Pasadena, CA 91125 (astuart@ caltech.edu).

$\S$ Mathematics Institute, University of Warwick, Coventry CV4 7AL, UK, and RICAM, Austrian Academy of Sciences, 4040 Linz, Austria (m.wolfram@warwick.ac.uk).
} 
the macroscopic model is still an open problem in certain scaling regimes. In this case more general interactions, as proposed in $[1,8,21,25]$, can be considered. In this paper we focus on mean-field interactions only. Here individual dynamics is influenced by the averaged behavior of the crowd, which can be characterized by the average velocity, flow, or density. Often, for example, in high density regimes, individual dynamics has little influence on the overall flow. For this reason averaged quantities are of specific interest to characterize the crowd behavior. While individual-based models depend on a large number of parameters, which allow a detailed description of individual trajectories, their identification and estimation are extremely challenging and computationally costly. Hence we focus on the identification of macroscopic relations from individual trajectories in the following.

An important and commonly used macroscopic relation is the so-called fundamental diagram. The fundamental diagram relates an averaged observed pedestrian density to either the measured velocity or outflow. It is a well-established characteristic quantity in vehicle traffic flow theory (see [30]) but also plays an important role in pedestrian dynamics. Examples of its use include the quantification of the capacity of pedestrian facilities or the evaluation of pedestrian models; see [39]. To understand the latter we may use trajectories which are collected in controlled experiments. These experiments are conducted for various conditions - different domains (corridors, junctions, etc.), uni- and bidirectional flows, varying inflow and outflow conditions, in addition to considering the effects of diverse social and cultural backgrounds; see, for example, [5]. In an experiment, trajectory recordings usually start once the experiment has equilibrated. The collected video or sensor data is used to extract individual trajectory data. More recently, also images from motion sensing cameras, such as Kinect, have been used (see [10]) to collect individual trajectories in public spaces. The fundamental diagram is then calculated by evaluating the individual trajectory data using suitable averaging techniques. In unidirectional flows different regimes can be observed, and at low densities pedestrians walk with a (maximum) velocity (denoted by $v_{\max }$ later on). Then the average speed decreases as the density increases. At a certain point the velocity approaches zero due to overcrowding. Later on, we refer to the density at which overcrowding occurs as $\rho_{\max }$. Different values for $\rho_{\max }$ and $v_{\max }$ can be found in the literature, ranging from 3.8 to 10 pedestrians per $\mathrm{m}^{2}$ for $\rho_{\max }$, and from $0.98 \mathrm{~m} / \mathrm{s}$ to $1.5 \mathrm{~m} / \mathrm{s}$ for $v_{\max }$. These deviations can be explained by the experimental setup and measurement techniques used, as well as psychological and cultural factors; cf. [7, 39, 43].

In this paper we consider a unidirectional flow in a corridor as illustrated in Figure 1.1a. We assume that pedestrians enter the corridor on the left through $\Gamma_{i n}$ and exit on the right through $\Gamma_{\text {out }}$, with no possible entrance or exit at the walls $\Gamma_{N}$.

The evolution of the overall density $\rho=\rho(x, t)$ can be modeled by a nonlinear Fokker-Planck equation, with (nonlinear) mixed boundary conditions, describing the inflow and outflow at the entrance and exit. Here the drift term accounts for the nonlinear velocity's dependence on the density, as described by the fundamental diagram. The scaled density $\rho$ allows us to describe individual trajectories $X=X(t)$ as realizations of a generalized McKean-Vlasov process. This introduces a coupling between the (microscopic) trajectories $X$ and the (macroscopic) density $\rho$ of the system, with $X$ governed by a density dependent stochastic differential equation (SDE) (2.6) and $\rho$ governed by a Fokker-Planck-type partial differential equation (PDE) (2.1). It is important to note that the density $\rho$ is not a probability density function, since the total mass changes in time due to the inflow and outflow boundary conditions. Rather than directly employing the empirical density of the process to perform pa- 


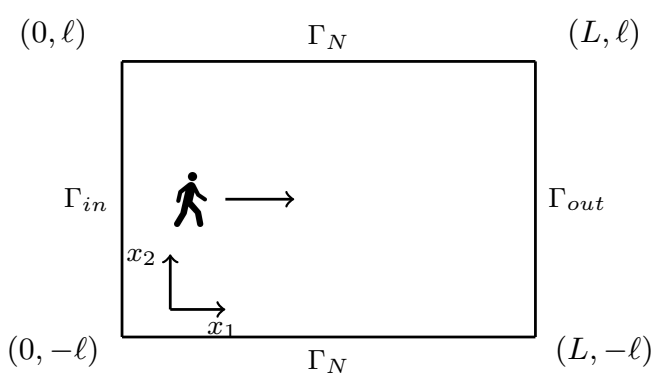

(a) Sketch of the domain.

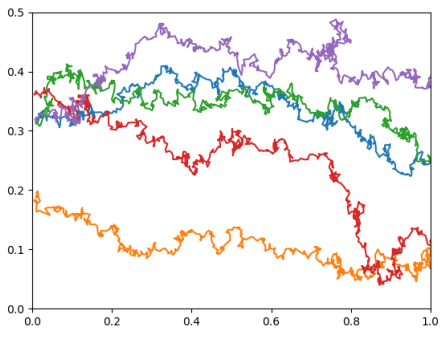

(b) Sample of five generated trajectories.

FIG. 1.1. Modeling setup considered.

rameter estimation, we instead try to learn parameters from individual trajectories whose guiding velocity depends on the density via the fundamental diagram; this avoids the difficult problem of creating smooth densities from collections of individual trajectories. We study the coupling between the PDE and the trajectories of each pedestrian with the goal of learning two parameters from individual trajectories of the resulting nonlinear Markov process: the maximum speed $v_{\max }$ and the maximum density $\rho_{\max }$. While there is some work concerning parameter estimation of a nonlinear Markov process, this is primarily focused on settings in which the data concerns macroscopic or mean properties [20]; see also the recent analyses of nonlinear Markov processes in $[17,18]$. In contrast we focus on learning from microscopic data; closely related work concerns the learning of (macroscopic) ocean properties from Lagrangian (microscopic) trajectories [2].

We will see that the maximum density $\rho_{\max }$ is not identifiable in our setup. The parameter $v_{\max }$ is learnable and will be determined using a maximum a posteriori (MAP) estimator as well as a fully Bayesian methodology. Estimates such as MAP (as well as the related maximum likelihood estimator (MLE)) are asymptotically unbiased and asymptotically normal as long as the path observations are sufficiently long (i.e., observe the evolution of the generalized McKean-Vlasov process for $T \rightarrow$ $\infty)[28,36]$. However, since individuals leave the domain after a finite time, we use multiple trajectories and show that this leads to properties similar to those found when observing one very long trajectory of an SDE.

The considered mathematical model is rather simplistic from a modeling perspective. At the moment we do not account for many known features of individual behavior, such as collision avoidance or small group dynamics. However, the proposed setup is an appropriate model for the density of the process and still mathematically tractable. The developed framework will allow us to extend and generalize our parameter learning approach to more complicated models, geometries, and situations in the future. Furthermore we observe that the SDE model considered describes individual motion only. The (Brownian) noise models the tendency of individuals to not move along completely straight lines. However, it does not correspond to measurement errors in experiments. Including such errors would involve an extra equation for the trajectory observations and requires the use of filtering methods. This problem could be considered in the future; however, it is likely that the model error itself is larger than the observation error in such situations, and investment in refining the model is 
arguably more important than accounting for noise in the trajectory data.

This paper builds a systematic methodology to identify parameters of interest in systems which are modeled by SDEs which depend on their own density. The methodology is robust to improvements in the model and can be extended to more complex sensing of individual trajectories and to the incorporation of noise into the trajectory data. This approach can also be used to identify functions instead of parameters and quantify uncertainty in our estimates. There has been an increasing interest in developing connections between trajectory data and mathematical models; see, for example, [4]. However, in our approach the likelihood is constructed explicitly, using properties of stochastic differential equations, allowing for fully Bayesian inference, and avoiding the need for approximate Bayesian inference, as employed in the recent work by Bode, Chraibi, and Holl [4].

Our main contributions are the following:

- setup of parameter estimation methodologies for SDEs which depend on their own density, with trajectories confined to bounded domains via boundary conditions;

- discussion of identifiability in the considered model of pedestrian dynamics;

- development of numerical methods which allow for parameter estimation via optimization and for uncertainty quantification via a Bayesian approach;

- analyzing the impact of flow conditions (inflow vs. outflow rate, transient vs. steady state profiles) on the parameter estimation;

- comparison between transient and steady state density regimes, providing novel insights about the applicability of the fundamental diagram in transient regimes (we recall that the fundamental diagram is obtained from equilibrated experimental data).

The paper is organized as follows. We start by introducing the underlying mathematical models in section 2. Next we present analytic results for the PDE and SDE models in section 3 and introduce the parameter identification problem in section 4. The computational complexity of the considered identification problem requires efficient and robust numerical solvers for the PDE and SDE models. We discuss in section 5 these solvers as well as the optimization methodologies used. We conclude in section 6 by discussing the identifiability of $v_{\max }$ in various density regimes and for different experimental setups with numerous computational experiments. We present our conclusions in section 7. Further details on the analysis of the PDE will be given in the appendix.

2. The microscopic and the macroscopic models. We consider the unidirectional flow of a large number of pedestrians moving in a corridor $\Omega=\left\{\left(x_{1}, x_{2}\right) \in\right.$ $[0, L] \times[-\ell, \ell]\}$ as illustrated in Figure 1.1a. On the macroscopic level the evolution of the density $\rho(x, t)$ can be described by a nonlinear Fokker-Planck (FP) equation,

$$
\partial_{t} \rho(x, t)=\operatorname{div}(\Sigma \nabla \rho(x, t)-\rho(x, t) F(\rho)),
$$

where the diffusion matrix is of the form $\Sigma=\operatorname{diag}\left(\sigma_{1}^{2}, \sigma_{2}^{2}\right)$. The diffusion accounts for the tendency of pedestrians to not move along perfectly straight lines, and its structure allows for different diffusivities in the vertical and horizontal directions. Since individuals move from the left to the right, we choose a convective field $F: \mathbb{R} \mapsto$ $\mathbb{R}^{2}$ of the form

$$
F(\rho)=f(\rho) e_{1}, \text { where } f: \mathbb{R}^{+} \mapsto \mathbb{R}^{+} \text {and } e_{1}=\left(\begin{array}{l}
1 \\
0
\end{array}\right) .
$$

Copyright (C) by SIAM. Unauthorized reproduction of this article is prohibited. 
This convective field depends on density only, and will be evaluated pointwise to drive individual trajectories. ${ }^{1}$ We choose the velocity as

$$
f(\rho)=v_{\max }\left(1-\frac{\rho}{\rho_{\max }}\right),
$$

which corresponds to the density-flow relation observed in the fundamental diagram in traffic flow and pedestrian dynamics. Thus the model states that all individuals want to move at a maximum speed $v_{\max }$ in the absence of other pedestrians, and the actual velocity decreases linearly from this value with the density $\rho$ evaluated locally. We assume that the corridor is initially empty, that is, $\rho(x, 0)=0$ for all $x \in \Omega$. Individuals enter at a certain rate $a$ on the left through the entrance $\Gamma_{i n}$ and leave on the right through the exit $\Gamma_{\text {out }}$ at a rate $b$. On the rest of the boundary we impose no flux boundary conditions. Let $j=-\Sigma \nabla \rho+F(\rho) \rho$ denote the flux. Then the corresponding boundary conditions are given by

$$
\begin{array}{ll}
j \cdot n=-a\left(\rho_{\max }-\rho\right) & \text { for all }\left(x_{1}, x_{2}\right) \in \Gamma_{i n}, \\
j \cdot n=b \rho & \text { for all }\left(x_{1}, x_{2}\right) \in \Gamma_{\text {out }}, \\
j \cdot n=0 & \text { for all }\left(x_{1}, x_{2}\right) \in \Gamma_{N},
\end{array}
$$

where

$$
\begin{aligned}
& \Gamma_{\text {in }}=\left\{\left(x_{1}, x_{2}\right) \in \Omega: x_{1}=0\right\}, \Gamma_{\text {out }}=\left\{\left(x_{1}, x_{2}\right) \in \Omega: x_{1}=L\right\} \\
& \Gamma_{N}=\left\{\left(x_{1}, x_{2}\right) \in \Omega: x_{2}= \pm \ell\right\}
\end{aligned}
$$

and $n$ denotes the unit outer normal vector. Note that the inflow condition $(2.4 \mathrm{a})$ includes the additional factor $\left(\rho_{\max }-\rho\right)$ due to volume exclusion. This prefactor arises in the formal limit, when particles are only allowed to enter or move to a certain position if enough physical space is available; see [42]. We note that $a$ and $b$ are rates of entrance and exit in the domain. Balancing the left- and right-hand sides of (2.4a) and (2.4b) gives us their units - both $a$ and $b$ are in $m / s$. A small modification of the maximum principle calculations in [6] shows that it is sufficient for the problem to be well-posed that $0 \leq a, b \leq v_{\max }$.

In the following we will see that trajectory data generated by the model (2.1) is independent of the parameter $\rho_{\max }$. Let $\tilde{\rho}=\frac{\rho}{\rho_{\max }}$; then $(2.1)$ can be rescaled as

$$
\partial_{t} \tilde{\rho}=\operatorname{div}(\Sigma \nabla \tilde{\rho}-\tilde{\rho} \tilde{F}(\tilde{\rho}))
$$

where

$$
\tilde{F}(\tilde{\rho})=\tilde{f}(\tilde{\rho}) e_{1}, \quad \tilde{f}(\tilde{\rho})=v_{\max }(1-\tilde{\rho}) .
$$

The system is supplemented with the boundary conditions

$$
\begin{aligned}
& \tilde{j} \cdot n=-a(1-\tilde{\rho}) \quad \text { for all }\left(x_{1}, x_{2}\right) \in \Gamma_{i n}, \\
& \tilde{j} \cdot n=b \tilde{\rho} \quad \text { for all }\left(x_{1}, x_{2}\right) \in \Gamma_{\text {out }} \text {, } \\
& \tilde{j} \cdot n=0 \quad \text { for all }\left(x_{1}, x_{2}\right) \in \Gamma_{N} \text {. }
\end{aligned}
$$

\footnotetext{
${ }^{1}$ Later we will replace $e_{1}$, the direction of motion, by a gradient vector field to allow for more complex domains such as bottlenecks; this formulation will be used in the existence and uniqueness proof of the PDE as well as the bottleneck example presented in section 6 .
} 
Here the scaled flux is given by $\tilde{j}=-\Sigma \nabla \tilde{\rho}+v_{\max }(1-\tilde{\rho}) \tilde{\rho} e_{1}$. We see that the maximum density $\rho_{\max }$ is not present in the scaled formulation. Furthermore the convective field $F$, which governs individual trajectories, is given by (2.2), (2.3) and can be expressed entirely in terms of $\tilde{\rho}$, with no reference to $\rho_{\max }$.

Our stated aim is to identify $v_{\max }$ and $\rho_{\max }$ in $f(\rho)(2.3)$ using individual trajectories. However, the preceding argument shows that the parameter $\rho_{\max }$ does not influence the SDE for trajectories. Since our parameter inference is based only on these trajectories, this means that $\rho_{\max }$ cannot be learned from the data available to us. Hence we make the following remark.

Remark 2.1. The parameter $\rho_{\max }$ cannot be identified within our adopted microscopic-macroscopic data-model framework. This limitation is not caused by the identification methodologies proposed, but rather by the invariance of the PDE-SDE model to scaling in $\rho$ and the fact that the measured trajectory data is independent of the value of $\rho_{\max }$. Indeed we initially conducted numerical experiments using the unscaled model, which led to the understanding that $\rho_{\max }$ is not identifiable. Similar identifiability issues are well-known in the literature relating to inference for diffusion processes; see, for example, the paper [37], in which it is shown that the quadratic variation of sample paths cannot contain information about time-rescaling.

For this reason the rest of this paper is based only on the scaled FP equation for $\tilde{\rho}$. However, we wish to drop the $\sim$ notation for ease of presentation. This corresponds to using (2.1)-(2.4) with $\rho_{\max }=1$. Since the preceding arguments show that $\rho_{\max }$ is not identifiable from trajectory data, making any specific choice of $\rho_{\max }$, including the value 1 , will not affect the inference for $v_{\max }$. The parameter $\rho_{\max }$ thus plays no further part in the paper.

Our focus, then, is on identification of $v_{\max }$. We will use the scaled FP equation (2.5) in the following, which coincides with the system (2.1)-(2.4) when setting $\rho_{\max }=$ 1.

The existence of steady states as well as the different stationary regimes - the socalled influx limited, outflux limited, and maximum current regimes - are discussed by Burger and Pietschmann in [6]. We present corresponding existence results for the time dependent problem in section 3 . Note that the steady state as well as the time dependent solutions of (2.5) satisfy $0 \leq \rho \leq 1$, which ensures that $f$ stays nonnegative for all $x \in \Omega$ and times $t>0$. This also allows us to define individual trajectories as realizations of the following generalized McKean-Vlasov equation:

$$
d X(t)=F(\rho(X(t), t)) d t+\sqrt{2 \Sigma} d W(t),
$$

where $W(\cdot)$ is a unit Brownian motion and $\rho$ solves (2.5). These realizations correspond to individual trajectories as we see in Figure 1.1b.

In the presented PDE model the boundary conditions drive the dynamics of the process. Hence they need to be included at the SDE level as well if we wish for self-consistency. Boundary conditions for SDEs are a delicate issue, and a general existence theory is not available in closed domains. It is beyond the scope of this paper to state the SDE problem in a rigorous manner (e.g., using local time [41]). However, it is important to implement the boundary conditions in a consistent way with the PDE, and we discuss their implementation in more detail in section 5.2. Once this is done we note that the scaled FP equation is self-consistent with the forward Kolmogorov equation for this SDE. Indeed any sufficiently regular solution $\rho$ of the FP equation (2.5) can be used as a drift in (2.6). The corresponding FP equation is then a linear PDE with the same solution as the nonlinear PDE (2.5). See 
[32, Thm. 1], [16, Thm. 7.3.1], [13, 19] for theory relating to such PDEs in related settings.

3. Analysis of the model. In this section we discuss the analysis of the generalized McKean-Vlasov SDE (2.6) as well as the rescaled FP equation (2.5). We start with the existence and regularity results for the FP equation, as they ensure the well-posedness of the corresponding generalized McKean-Vlasov process. We reemphasize the important fact, discussed prior to Remark 2.1, that (2.5) is identical to $(2.1)-(2.4)$, with $\rho_{\max }=1$.
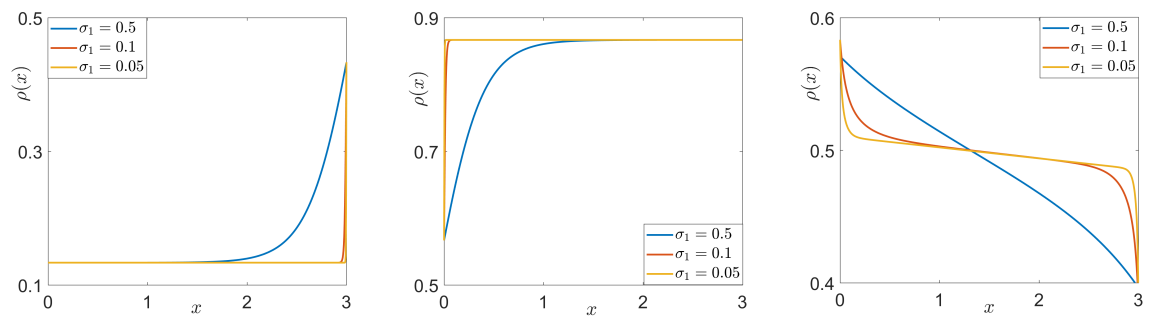

FIG. 3.1. Examples of the steady states of the FP equation in $1 D$ for $\sigma=0.5,0.1,0.05$ and (left) the influx limited phase $-a=0.2, b=0.4$, (center) the outflux limited phase $-a=0.4, b=0.2$, and (right) the maximal current phase $-a=0.9, b=0.975$.

3.1. Analysis of the Fokker-Planck equation. The nonlinear boundary conditions (2.5c) and (2.5d) strongly influence the time dependent and steady state solutions. The relation of the maximum velocity $v_{\max }$ to the inflow and outflow parameters $a$ and $b$ defines different stationary regimes, in which boundary layers arise at the entrance and/or the exit.

The following regimes were introduced in [42] and were characterized and analyzed by Burger and Pietschmann in [6]:

(1) Influx limited phase in the case $a<b$ and $\min (a, b)<\frac{v_{\max }}{2}$. We observe an asymptotically low density $\left(\rho<\frac{1}{2}\right)$ and a boundary layer at the exit.

(2) Outflux limited phase for $a>b$ and $\min (a, b)<\frac{v_{\max }}{2}$. This creates an asymptotically high density $\left(\rho>\frac{1}{2}\right)$ and a boundary layer at the entrance.

(3) Maximal current phase if $a, b \geq \frac{v_{\max }}{2}$. Here we observe an asymptotic density, with $\rho \approx \frac{1}{2}$ in most of the domain when $\sigma$ is small, and boundary layers at the entrance and exit. In the case $a=b=\frac{v_{\max }}{2}$, the unique steady state is given by $\rho(x)=\frac{1}{2}$.

Figure 3.1 illustrates the different regimes in 1D in the case of a corridor of length $L=3, v_{\max }=1.5$, and different values of $\sigma=\sigma_{1}$. We observe the predicted boundary layers at the entrance and/or exit, whose width is determined by the diffusivity $\sigma$. The existence of at least one steady state solution under suitable assumptions on the inflow and outflow boundary conditions as well as a more general regular potential was proven in [6]. Note that these steady state profiles are unique in 1D.

In the following we state and discuss the respective existence result for the time dependent problem. The proof uses techniques similar to those in [6] and can be found in the appendix. Without loss of generality we assume $\sigma_{1}=\sigma_{2}=1$ and $v_{\max }=1$. Furthermore, we consider the more general case in which $e_{1}$ is replaced by $\nabla V$ in (2.2). We make the following assumptions:

(A1) $\Omega \subset \mathbb{R}^{n}$ bounded, $n=1,2,3$, with boundary $\partial \Omega \in C^{2}$.

(A2) $0 \leq a, b \leq 1$, and $\min \{a, b\}<1$. 
(A3) $V \in W^{1, \infty}(\Omega)$, and $\Delta V=0$.

Assumption (A1) actually excludes domains like the corridor shown in Figure 1.1a. We still employ the boundary conditions $(2.5 \mathrm{c})-(2.5 \mathrm{e})$, simply assuming that the union of (the disjoint) $\Gamma_{\text {in }}, \Gamma_{\text {out }}$, and $\Gamma_{N}$ comprises the entire boundary $\partial \Omega$. One could consider a rectangular domain with rounded off corners instead, but since we did not observe any issues in the computational experiments with corners, we retained them for a more realistic simulation setting.

We seek weak solutions in the set

$$
\mathcal{S}=\{\rho \in \mathbb{R}: 0 \leq \rho \leq 1\},
$$

and refer to its interior as $\mathcal{S}^{0}$. Then the weak solution $\rho: \Omega \times[0, T] \rightarrow \mathcal{S}$ satisfies

$$
\int_{0}^{T}\left[\left\langle\partial_{t} \rho, \varphi\right\rangle_{H^{-1}, H^{1}}-\int_{\Omega} j \cdot \nabla \varphi d x-a \int_{\Gamma_{\text {in }}}(1-\rho) \varphi d s+b \int_{\Gamma_{\text {out }}} \rho \varphi d s\right] d t=0
$$

for all test functions $\varphi \in L^{2}\left(0, T ; H^{1}(\Omega)\right)$.

THEOREM 3.1. Let assumptions (A1)-(A3) be satisfied. Let $\rho_{0}: \Omega \rightarrow \mathcal{S}^{0}$ be a measurable function such that the initial entropy $E$ defined by (A.1) satisfies $E\left(\rho_{0}\right)<$ $\infty$. Then there exists a weak solution to system (2.5) in the sense of (3.2) satisfying

$$
\begin{aligned}
\partial_{t} \rho & \in L^{2}\left(0, T ; H^{1}(\Omega)^{*}\right), \\
\rho & \in L^{2}\left(0, T ; H^{1}(\Omega)\right) .
\end{aligned}
$$

In the preceding theorem, the star denotes the dual operation. The existence proof as well as improved regularity results can be found in the appendix.

3.2. Analytic considerations on the generalized McKean-Vlasov equation. The analytic results for the FP equation (2.5) would allow us to prove existence and uniqueness of strong solutions for $(2.6)$ in $\mathbb{R}^{2}$. However, since we consider the setup of a corridor with a mix of boundary conditions (reflected in the walls, and partially reflected in the entrance and exit), standard results are not applicable. The only available results for SDEs with boundaries are slight generalizations of Skorokhod's problem [41] and can only be used for SDEs in the half plane. We are not aware of any results for more complicated geometries. However, our numerical experiments show that a careful discretization of the process, and in particular treatment of trajectories impinging on the boundary of $\Omega$, yields bounded and continuous solutions of (2.6).

4. Parameter estimation. In this section we introduce the parameter estimation framework for SDEs which depend on their own density. We will use a Bayesian approach based on sampling from a posterior distribution, and confirm our results with the computation of a maximum a posteriori (MAP) estimator. We recall that our initial goal was to estimate both $\rho_{\max }$ and $v_{\max }$. However, as we have seen, determination of $\rho_{\max }$ is not possible using the current setting, and only determination of $v_{\max }$ is feasible.

We estimate $v:=v_{\max }$ from a collection of sample paths $\left\{X_{i}(t)\right\}_{t \in[0, T]}^{i=1, \ldots, J}$ which are realizations of the McKean-Vlasov equation (2.6). In what follows we parameterize $f(\cdot)$ by $v$ and write $f(\cdot ; v)$. For ease of presentation, we discuss the estimation from a single trajectory before generalizing it for multiple trajectories. 
Let $X=X(t)$ be a realization of (2.6). Throughout the remainder of the paper we use the notation

$$
|\cdot|_{A}=\left|A^{-\frac{1}{2}} \cdot\right|
$$

where $|\cdot|$ is the Euclidean norm and $A$ any positive-definite symmetric matrix; a corresponding inner-product may be defined by polarization. Since $\dot{W}$ is white, it is intuitive that finding the best value of the parameter $v$, given an observation of a trajectory $X$, corresponds to minimizing the function

$$
\Phi(v ; X)=\frac{1}{4} \int_{0}^{T}|\dot{X}-F(\rho(X(t), t) ; v)|_{\Sigma}^{2}
$$

over all possible values of $v$. However, the function $\Phi(v ; \cdot)$ is almost surely infinite. In order to avoid this problem, we note that we can write

$$
\Phi(v ; X)=\Psi(v ; X)+\frac{1}{4} \int_{0}^{T}|\dot{X}|^{2} d t
$$

where the function $\Psi$ is defined as

$$
\Psi(v ; X)=\frac{1}{4} \int_{0}^{T}\left(|F(\rho(X(t), t) ; v)|_{\Sigma}^{2} d t-2\langle F(\rho(X(t), t) ; v), d X(t)\rangle_{\Sigma}\right) .
$$

Note that the last term in $\Psi$ is an Itô stochastic integral. It is preferable to perform the parameter estimation using $\Psi$ instead of $\Phi$, since the latter is infinite almost surely, while the former is finite almost surely, and since $\int_{0}^{T}|\dot{X}|^{2} d t$ does not depend explicitly on $v$ both cases yield the same results when applied in discretized form.

We will also add prior information and perform the parametric estimation of $v_{\max }$ by minimizing

$$
\mathcal{J}(v ; X):=\Psi(v ; X)+\frac{1}{2 c}|v-m|^{2},
$$

subject to the constraint that $v$ is positive. The parameters $m$ and $c$ correspond, respectively, to a prior estimate of the velocity and the variance associated with this estimate. This optimization problem will now be derived through a detailed description of the Bayesian formulation of inversion. Specifically we define precisely the Bayesian problem for the posterior distribution of $v$ given a trajectory $\{X(t)\}_{t \in[0, T]}$; this posterior distribution is maximized at the constrained minimization problem just defined.

In this Bayesian context the functional $\mathcal{J}$ can be interpreted as follows. The first term measures the misfit between the observed trajectory and the predicted density regime obtained from the $\mathrm{FP}$ equation. The second term is a regularization term, which is weighted by prior knowledge on $v$. In particular, we assume that $v$ is normally distributed with mean $m$ and variance $c$, conditioned to be positive. The probabilistic interpretation of $\mathcal{J}$ is as follows. The function $\exp (-\mathcal{J}(v ; X)) \mathbb{1}(v>0)$, appropriately normalized, is a probability distribution and in fact the posterior distribution, $\mathbb{P}(v \mid X)$, of $v$ given a realization $X$. This conditional distribution can be obtained, up to a normalization constant which is independent of $v$, by computing the joint probability of the process $X$ and the random variable $v$, which is given by

$$
\mathbb{P}(X, v)=\mathbb{P}(v \mid X) \mathbb{P}(X) ;
$$


thus

$$
\mathbb{P}(v \mid X) \propto \mathbb{P}(X, v)
$$

where the constant of proportionality is independent of $v$. Consider the probability measure $\lambda=\mathbb{Q} \otimes \operatorname{Leb}(\mathbb{R})$, where $\mathbb{Q}$ is the law of the Brownian motion driving the process and $\operatorname{Leb}(\mathbb{R})$ is the Lebesgue measure in $\mathbb{R}$. Lemma 5.3 in [22] states that

$$
\frac{d \mathbb{P}}{d \mathbb{\lambda}}(X, v)=\frac{d \mathbb{P}}{d \mathbb{Q}}(X \mid v) \pi_{0}(v),
$$

where $\pi_{0}$ is the prior Lebesgue density for $v$. Using Girsanov's theorem [14, 33], we see that

$$
\frac{d \mathbb{P}}{d \mathbb{Q}}(X \mid v)=\exp (-\Psi(v ; X))
$$

where $\Psi$ is defined in (4.3). If we choose $\pi_{0}(d v)=\mathbb{1}(v>0) N(m, c)(d v)$, which ensures that the velocity is almost surely positive both a priori and hence a posteriori, then (4.5) gives

$$
\mathbb{P}(v \mid X) \propto \exp \left(-\Psi(v ; X)-\frac{1}{2 c}|v-m|^{2}\right) \mathbb{1}(v>0),
$$

which is exactly $\exp (-\mathcal{J}(v ; X)) \mathbb{1}(v>0)$.

We can either sample from the probability distribution given by (4.8) (the Bayesian approach) or we can simply minimize the negative log likelihood of the data $\mathcal{J}(v ; X)$, subject to $v$ being positive. While the fully Bayesian framework allows us to quantify the uncertainty of our estimate, the minimization approach only gives a single point estimator. It is, however, much cheaper to simply minimize. In the following sections we will compare the results of the two approaches by sampling from the posterior distribution using the preconditioned Crank-Nicolson (pCN) algorithm, and minimizing the function $\mathcal{J}$ using the Nelder-Mead algorithm. Both methods are derivative-free, so they do not involve any extra function evaluation due to computing derivatives. We note that other derivative-free samplers or optimizers could be used.

The accuracy of the aforementioned estimators can be expected to improve with the lifetime of the observations [36]. Since in our setting trajectories terminate when they exit the domain, we are only able to observe them for a finite time. We will see that using multiple trajectories to estimate parameters has similar desirable properties to observing a single trajectory of a standard (boundaryless) SDE over a long time horizon.

We denote a family of such trajectories by $X_{j}(t), j=1, \ldots, J$. We assume that each trajectory $X_{j}$ starts at $t_{0}^{j}$ with $X_{j}\left(t_{0}^{j}\right)=X_{j}^{0}$ and exits at time $t=t_{f}^{j}$. We set $F\left(\rho\left(X_{j}(t), t\right) ; v_{\max }\right)=0$ for $t \notin\left[t_{0}^{j}, t_{f}^{j}\right]$, which allows us to define

$$
\psi_{j}\left(v ; X_{j}(t)\right)=\frac{1}{4} \int_{0}^{T}\left(\left|F\left(\rho\left(X_{j}(t), t\right) ; v\right)\right|_{\Sigma}^{2} d t-2\left\langle F\left(\rho\left(X_{j}(t), t\right) ; v\right), d X_{j}(t)\right\rangle_{\Sigma}\right) .
$$

Then we can sum the misfit of all trajectories and obtain

$$
\Psi_{J}\left(v ; X_{1}, \ldots, X_{J}\right)=\sum_{j=1}^{J} \psi_{j}\left(v ; X_{j}\right) .
$$

Copyright $@$ by SIAM. Unauthorized reproduction of this article is prohibited. 
Since all $\psi_{j}$ are independent, we can apply the previous argument, replacing $\Psi(v, X)$ by (4.9). The averaging over all the trajectories has the same effect as considering large values of $T$ for a single trajectory. This can be shown formally, using techniques similar to those used in [28] for a single trajectory.

It could be of interest to also estimate the diffusion coefficient matrix $\Sigma$. Estimating constant diffusion coefficients for sufficiently frequent observations is, in theory, a well-understood problem; see $[26,36]$. However our model is likely to be inconsistent with the data at small scales, and so it is important to appreciate that estimation of $\Sigma$ might well be a nontrivial problem, requiring techniques such as those introduced in [34].

5. Computational methods. In this section we discuss the discretization of the nonlinear PDE (2.5) and SDE (2.6) before continuing with parameter estimation in the following section. Note that the parameter estimation is computationally costly in the sense that it involves the solution of a PDE in every iteration of the sampling and optimization algorithms. Motivated by relevant experimental settings (noting that data collection starts after the experiment is equilibrated), we explore the effect of using both stationary and transient density regimes on the quality of our estimates. Using steady state density profiles also has the advantage of a lower computational cost. However, we will show that the steady state regimes do not produce consistent estimates across all parameter regimes, an effect which is mitigated by using (the more expensive) time dependent density-based estimation.

5.1. The time dependent and steady state Fokker-Planck solvers. The time dependent solver is based on the decomposition of (2.5a) into a diffusive and a convective part. For the nonlinear convective operator an upwind discontinuous Galerkin (DG) method and an explicit time integration scheme are used. For the discretization of the linear diffusive part we use a hybrid discontinuous Galerkin (HDG) method and an implicit time discretization. This additive time splitting, also known as the implicit-explicit (IMEX) method, allows us to treat the stiff diffusive term implicitly, while the nonlinear nonstiff hyperbolic problem is solved explicitly; see [3]. The IMEX scheme allows for larger time steps, while the (H)DG discretization ensures stability and parallelizability.

In some cases we employ 1D steady state profiles $\rho_{s}=\rho_{s}(x)$ (extended as constants in the orthogonal direction), and these are calculated using an $H^{1}$ conforming finite element discretization. We use a damped Newton method to solve the resulting nonlinear system, with an initial guess depending on the relation of $a$ and $b$. Both solvers use the finite element software package Netgen/NGSolve; see [38].

5.2. SDE solver and trajectory generation. We approximate all trajectories of (2.6) by using the explicit Euler-Maruyama scheme [24], which is a forward Euler time-stepping method. It consists of defining the approximation $X_{k} \approx X\left(t_{k}\right):=$ $X(k \Delta t)$ and updating the individual position as

$$
X_{k+1}=X_{k}+F\left(\rho\left(X_{k}, t_{k}\right)\right) \Delta t+\sqrt{2 \Sigma \Delta t} \xi_{k},
$$

where $\xi_{k} \sim N(0,1)$ and $\rho$ is the solution to the FP equation (which we advance in time simultaneously). To generate trajectories using the steady state profiles, we replace $\rho\left(X_{k}, t_{k}\right)$ by the steady state solution $\rho_{s}\left(X_{k}\right)$.

Since the diffusion coefficient is constant, the Euler-Maruyama scheme has strong order of convergence one [24], and we do not need to use more complicated timestepping methods. At the boundaries we implement individual rules consistent with 
TABLE 5.1

Implementation of the boundary conditions at the SDE level. The filled dot represents the current state, while the empty circles represent the possible position in the following time step, which is realized with probability $p$. Here we use the rescalings $P_{i n}=\sqrt{\pi \Delta t /\left(2 \sigma_{1}^{2}\right)} a\left(1-\rho\left(0, x_{2}\right)\right)$ and $P_{\text {out }}=\sqrt{\pi \Delta t / \sigma_{1}^{2}} b \rho\left(L, x_{2}\right)$ according to $[15]$.

\begin{tabular}{|c|c|c|c|}
\hline Boundary & $\mathrm{PDE}$ & $\mathrm{SDE}$ & Implementation \\
\hline$\Gamma_{N}$ & Neumann & Reflecting & \\
\hline $\begin{array}{c}\Gamma_{i n} \\
\text { (from outside) }\end{array}$ & Robin & Wait to get in & $\begin{array}{c}--\infty \\
p=P_{\text {in }}\end{array}$ \\
\hline $\begin{array}{c}\Gamma_{i n} \\
\text { (from inside) }\end{array}$ & Robin & Partially reflecting & $\begin{array}{c}p=P_{i n} \\
\text { back to } \Gamma_{i n}\end{array}$ \\
\hline$\Gamma_{\text {out }}$ & Robin & Partially reflecting & \\
\hline
\end{tabular}

(2.5). Singer et al. [40] and Erban and Chapman [15] derived appropriate scaled rates of entrance and exit for partially reflected boundaries in the half plane. These ensure that the Robin boundary conditions are satisfied by the corresponding probability densities. Even though it is not established that their results are directly applicable in the case of the more complex geometries we use here, we nonetheless follow their approach and implement the entrance and exit behavior as described in Table 5.1.

5.3. Derivative-free optimization - the Nelder-Mead algorithm. As detailed in section 4, we will use derivative-free methods for the minimization of (4.4), corresponding to finding the MAP estimator of the posterior probability distribution: the maximizer of (4.8). We choose the Nelder-Mead algorithm [31], which is a widely used nonlinear unconstrained optimization algorithm. It is a direct search method which is based on the function evaluation of $n+1$ points in an $n$-dimensional space. A rough outline of the method (see [9] for more details) is given in Algorithm 5.1. The number in boldface is the total number of function evaluations necessary if that particular step is performed. If the objective function is strictly convex, then the algorithm requires only 1 or 2 function evaluations per iteration. Furthermore, it converges in $1 \mathrm{D}$ for strictly convex functions [29]. In $2 \mathrm{D}$ only limited convergence results are known. We use the MATLAB implementation of the Nelder-Mead algorithm (via the function fminsearch) with a modification of $\mathcal{J}$ that penalizes negative values of $v$. We observe a large decrease of the objective function in the first few iterations, as reported in the literature for many other applications.

5.4. MCMC method - the pCN algorithm. The MAP estimator obtained with the Nelder-Mead algorithm does not provide us with any quantification of uncertainty on our estimates. In order to obtain such uncertainty estimates we sample from 


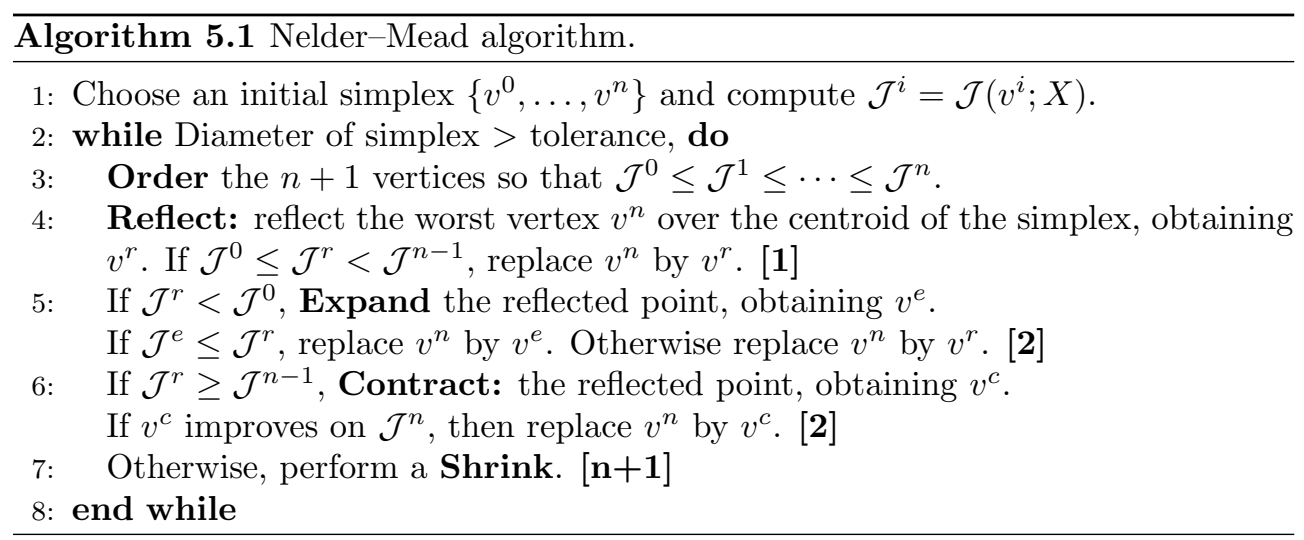

the posterior distribution given by (4.8). For this purpose we use the pCN method described in [11]; it is a general purpose method for sampling from any distribution which is formed as the product of a (not necessarily smooth) function $\mathfrak{f}$ and a Gaussian density and has the advantage that derivatives of $\mathfrak{f}$ are not required. In our problem this means that we avoid computing derivatives of $\Psi$. Looking forward, the method also has the potential to scale up to cases where the objective is to estimate the shape of $f(\rho)$ in (2.3) nonparametrically. Furthermore, the algorithm has a single tunable parameter $\beta$ which can be explored to maximize efficiency. Algorithm 5.2 gives the details.

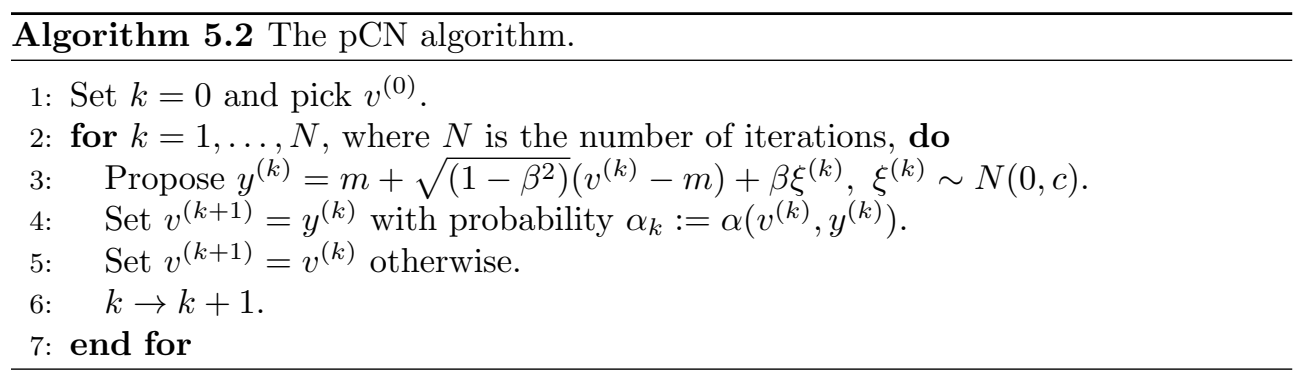

The acceptance probability is given by

$$
\alpha(v, y)=\min \{1, \exp (\Psi(v ; X)-\Psi(y ; X))\} \mathbb{1}(y>0) .
$$

Notice, in particular, that if $\Psi\left(y^{(k)} ; X\right) \leq \Psi\left(v^{(k)} ; X\right)$ and $y^{(k)}>0$, then the proposed move is accepted with probability one.

6. Numerical results. In this section we present and discuss numerical results based on the solvers and methodologies presented in section 5 . In particular we want to estimate $v_{\max }$ using multiple trajectories of the coupled SDE-FP system. These trajectories are generated using both the time dependent and the steady state solution of the FPE, and for different inflow and outflow conditions. The corresponding parameter estimation problem is then solved using the Nelder-Mead optimizer or the pCN Bayesian sampler. Our numerical simulations lead to the following conclusions:

1. The maximum speed $v_{\max }$ is learnable; see Figure 6.1.

2. Results are more accurate and less uncertain if the influx and outflux rate $a$ and $b$ differ considerably; see Figure 6.9. 
3. We do not obtain reliable estimates for all regimes using stationary density profiles; see Figure 6.5.

4. Estimates obtained from trajectories experiencing steady state densities are less uncertain than from time dependent ones (in the regimes where steady state estimation does give consistent results); see Figures 6.9 and 6.10 .

In order to test the parameter estimation methodology, we generate a collection of trajectories for a range of parameters which represent the different density regimes. To create this data we choose the following parameters for all trajectories: corridor length $L=3$, corridor width $2 \ell=0.5$, noise strength $\sigma_{1}=\sigma_{2}=\sigma_{0}=0.05$, and a final time $T=2$. The time steps in the SDE solver are set to $\Delta t_{S D E}=10^{-3}$. We assume that the true value for the maximum velocity is $v_{\max }=1.5 \mathrm{~m} / \mathrm{s}$, which is chosen close to values obtained from experiments. The final time is chosen sufficiently large to ensure that some individuals have the time to leave the domain. The size of the time steps corresponds to the setting in a high frequency regime. We generate trajectories using a time dependent density $\rho=\rho(x, t)$ satisfying $(2.5 \mathrm{a})$ as well as the steady state $\rho_{s}=\rho_{s}(x)$. Note that the latter choice is computationally less expensive and corresponds more closely to widely used experimental conditions, since data is typically collected once the pedestrian flow has equilibrated. We consider five sets of parameters which span the three steady state density regimes:

- outflux limited: $a=0.4, b=0.2$ and $a=0.45, b=0.4$

- influx limited: $a=0.2, b=0.4$ and $a=0.1, b=0.15$;

- maximal current: $a=0.9, b=0.975$.

Since the variance coefficients might not be known in practice, we will estimate the parameters using values for the diffusion coefficient which differ significantly from those present in the data; we take $\sigma_{1}=\sigma_{2}=\sigma=1$ in our algorithm. This corresponds to a form of model-mispecification and avoids committing an inverse crime [27].

6.1. Benchmarking. In the following, we present a first set of numerical results which confirm that the optimization and MCMC methodologies perform as expected. In particular, we will see that the value of the estimator for $v_{\max }$ is consistent across both methodologies, and is also independent of the various parameters used, such as time steps or spatial mesh, and the parameter $\beta$ of the $\mathrm{pCN}$ algorithm. Throughout this subsection we use $J=20$ trajectories.

Influence of the discretization. This test case is of particular interest with respect to the computational efficiency, since each iteration of either minimization algorithm requires one (or more) PDE solves. Therefore we wish to use time steps which are as large as possible as well as a mesh which is as coarse as possible. We first present numerical results related to the influence of the PDE time step on the value of the estimator for $v_{\max }$.

We run the pCN (and Nelder-Mead) algorithm using three different time steps for the PDE solve $-\Delta t=0.001, \Delta t=0.005$, and $\Delta t=0.01$ - and interpolate the obtained values to fit the time step of the SDE trajectories. We tested the influence of the time step in the maximal current regime with $a=0.9, b=0.975$. The posterior distributions for $v_{\max }$ as well as the corresponding prior distribution and the true value are depicted in Figure 6.1. These results are consistent with those obtained using the Nelder-Mead algorithm to compute the MAP estimator (successive iterations of the Nelder-Mead algorithm are depicted in the right panel with orange circles, and we point out that the number of iterations for the MAP estimator was scaled by a factor of approximately $10^{-3}$ for comparison). We observe that all the test cases produce similar results, with a slightly better agreement in the case of the finer time steps, 

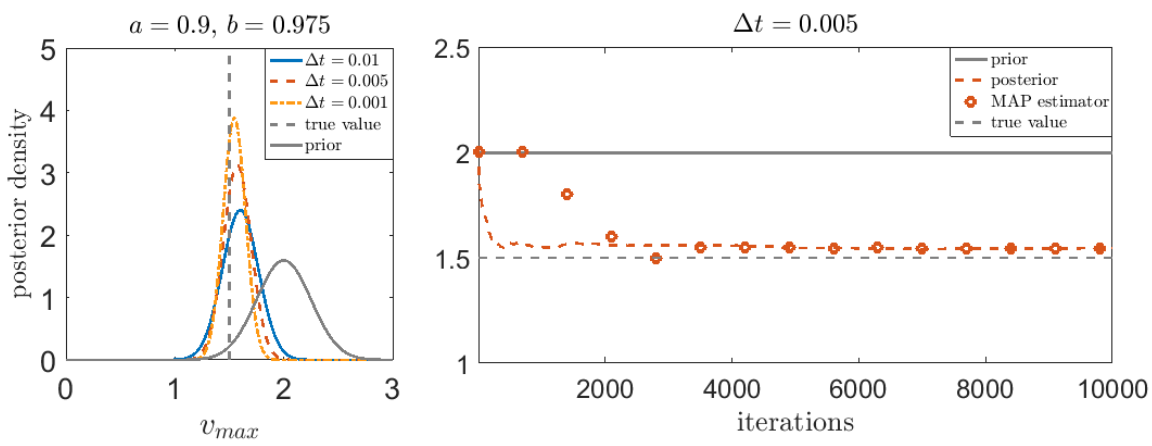

FIG. 6.1. Influence of the PDE time step on the estimator for a maximal current regime with $a=0.9, b=0.975$. Left: True value (gray dashed line), prior distribution (solid gray line), and posterior distributions for different PDE time steps. Right: Prior mean (solid gray line), true value (gray dashed line), posterior mean (orange dashed line), and MAP estimator iterations (orange circles) for $\Delta t=0.005$.

where the results are also less uncertain, since the posterior distribution has a smaller variance. For this reason, all the $\mathrm{pCN}$ and Nelder-Mead simulations presented below will be performed with a PDE time step of $\Delta t=0.005$.

We observe a similar agreement between the mean of the posterior distribution and the MAP estimator for all the results presented below; henceforth we present the posterior distribution only. We also point out that the MAP estimator (mode of the posterior distribution) is approximately the same as the running average (mean of the posterior distribution). This suggests that the posterior distribution is approximately Gaussian as is to be expected when the data is highly informative. Finally, we also tested the influence of the SDE time step (frequency of observations) and spatial mesh on the estimates, obtaining similarly good results.

Influence of the parameter $\beta$. The value of the estimator obtained using the $\mathrm{pCN}$ algorithm should be independent of the parameter $\beta$ since $\beta$ is a tunable parameter in the algorithm and not a parameter of the posterior distribution. In Figures 6.2 and 6.3 we plot the prior and posterior distributions for $v_{\max }$ in two influx limited regimes, $a=0.2, b=0.4$ (left panels) and $a=0.1, b=0.15$ (right panels). Figure 6.2 was obtained in a time dependent regime, while Figure 6.3 uses trajectories experiencing a steady state density. We observe in both figures that the posterior distribution is independent of the parameter $\beta$, as expected.

6.2. Influence of problem-specific parameters. In the following, we present our numerical results which confirm the conclusions presented at the beginning of this section. In particular, we will show that both approaches give consistent results with respect to the initial guess, prior mean and variance, and number of used trajectories. All the examples presented use $\beta=0.1$ and $J=20$ trajectories, except the case in which we vary the number of trajectories.

Influence of the initial guess and prior mean. We assume that $v_{\max }$ has prior distributions $N(m, c)$ with $m \in\{1,2\}$ and $c=0.25$ and test using two initial guesses $v=1$ and $v=2$. Figure 6.4 depicts the prior distribution (gray solid line), true value (gray dotted line), and the posterior distributions for each case tested. The MAP estimator agrees with the means of the posterior distributions for all cases. The left panel depicts the results for $m=1$, while the right panel has $m=2$. In each case we observe that the estimates are independent of the initial guess, and both prior 

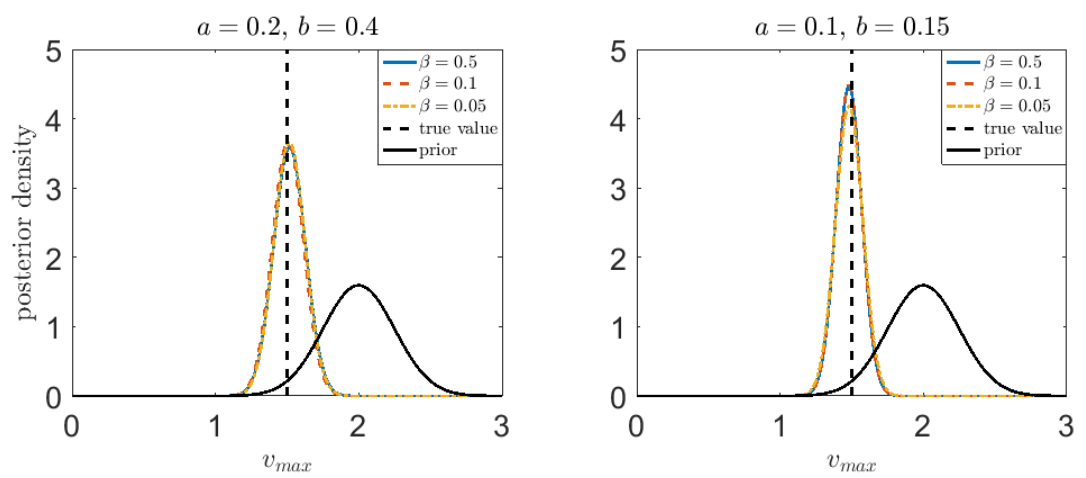

FiG. 6.2. Influence of the parameter $\beta$ on the estimator for two influx limited regimes and using the solution of the time dependent FP equation. Left: $a=0.2, b=0.4$. Right: $a=0.1, b=0.15$.
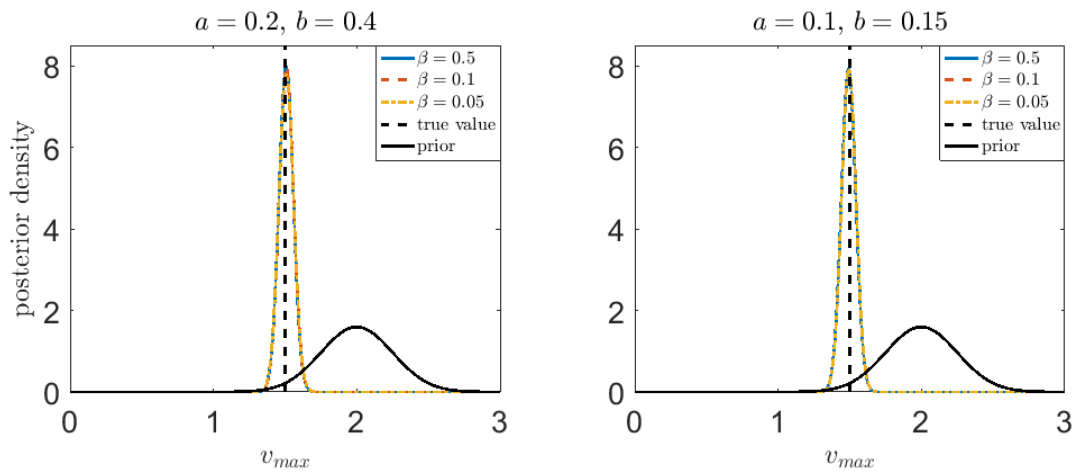

FIG. 6.3. Influence of the parameter $\beta$ in the posterior distribution of $v_{\max }$ guesses for influx limited regimes and using the solution of the steady state FP equation. Left: $a=0.2, b=0.4$. Right: $a=0.1, b=0.15$.

distributions produce similar results. Next we use trajectories generated with steady state density profiles.

Figure 6.5 shows the influence of initial guess and prior mean for three different regimes: an outflux regime with $a=0.4, b=0.2$ (left panel), an influx limited regime with $a=0.2, b=0.4$ (middle panel), and a maximal current regime with $a=$ $0.9, b=0.975$ (right panel). We use the same prior distributions and initial guesses as in Figure 6.4. We find perfect agreement between the Nelder-Mead and pCN estimators, and the Bayesian estimator has converged to the posterior distribution. The maximal current (right panel) and influx limited (left panel) regimes produce results similar to those of the corresponding time dependent case; however, in the outflux limited regime we can observe that the posterior distribution stays close to its prior distribution. The reason for this may be found in the trajectories used - in the outflux limited regimes, individuals experience very high densities and get "stuck." Since they cannot move, they do not experience the maximum velocity and carry little information about it. In the influx limited regimes, the overall density is smaller and does not influence the velocity that much. This can be seen from the depicted trajectories in Figure 6.6. Note that we do not observe similar problems using time dependent density profiles, since most trajectories experience significant velocities before the flow has equilibrated. 

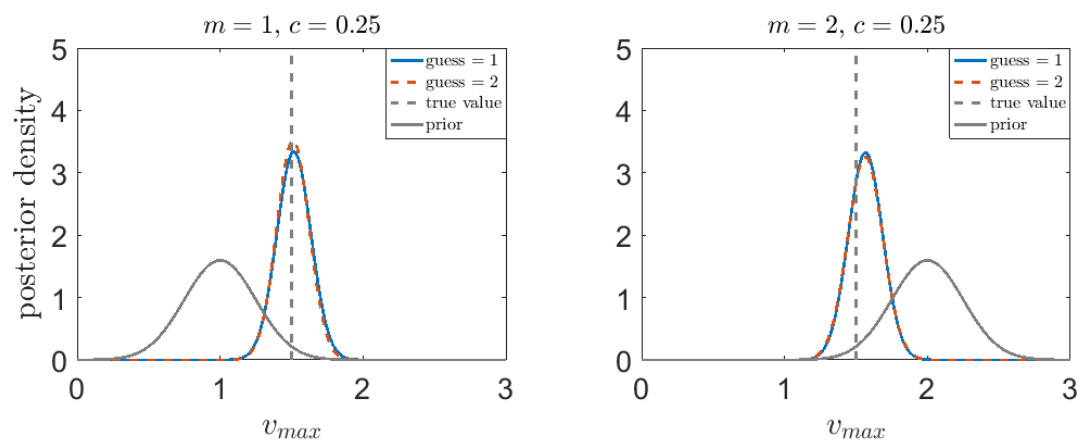

FIG. 6.4. Influence of prior distribution and initial guess for an outflux limited regime with $a=0.45, b=0.4$ using solutions of the time dependent FP equation. Left: Prior mean $m=1$. Right: Prior mean $m=2$. Both figures have $c=0.25$ and depict the prior distribution (solid gray line), true value (dashed gray line), and posterior distributions for initial guesses $v_{\max }=1,2$.
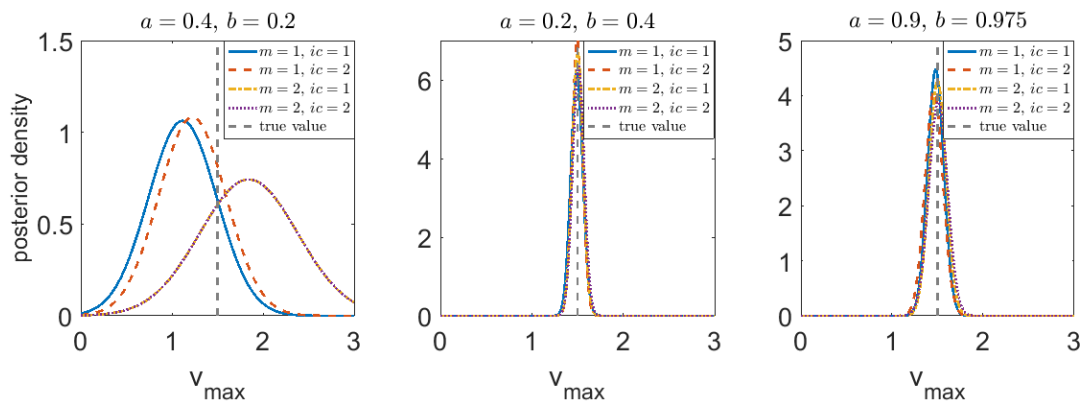

FIG. 6.5. Influence of prior distribution and initial guess for three different regimes using solutions of the steady state equation. Left: Outflux limited regime with $a=0.4, b=0.2$. Middle: Influx limited regime with $a=0.2, b=0.4$. Right: Maximal current regime with $a=0.9, b=0.975$.
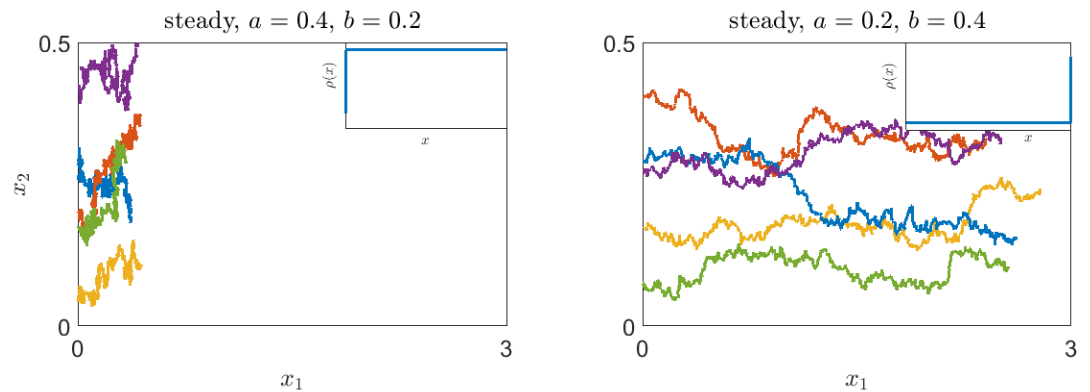

FIG. 6.6. Examples of five trajectories for steady state densities. Left: Outflux limited regime. Right: Influx limited regime.

Influence of the prior variance. The influence of the prior variance is depicted in Figure 6.7 for the time dependent case and in Figure 6.8 for steady states. Again, we test this for an outflux (left panels) and an influx (right panels) limited regime. As before, we observe that the estimate for $v_{\max }$ is accurate for both regimes in the time dependent case, except when the prior variance is too small, while for the steady state case we observe again that the outflux limited case produces a posterior distribution

Copyright (c) by SIAM. Unauthorized reproduction of this article is prohibited. 

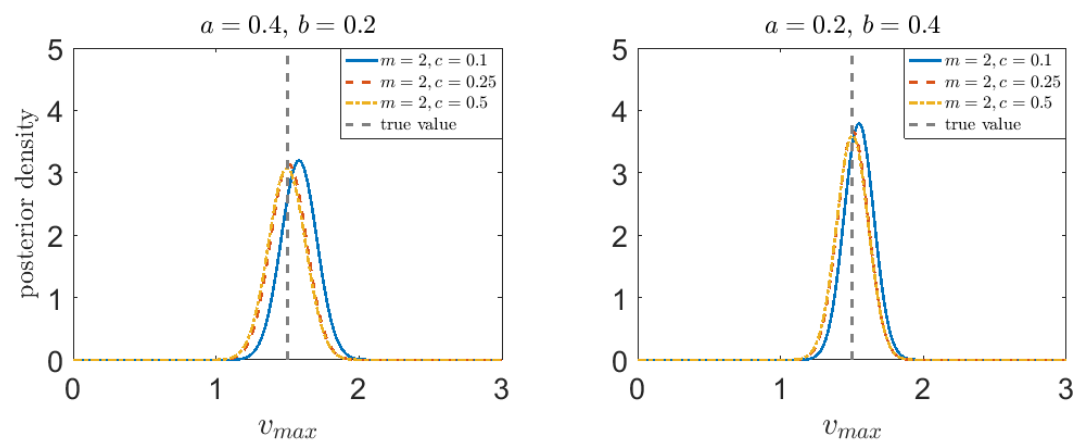

FIG. 6.7. Influence of prior variance for two different regimes using solutions of the time dependent FP equation. Left: Outflux limited regime with $a=0.4, b=0.2$. Right: Influx limited regime with $a=0.2, b=0.4$.
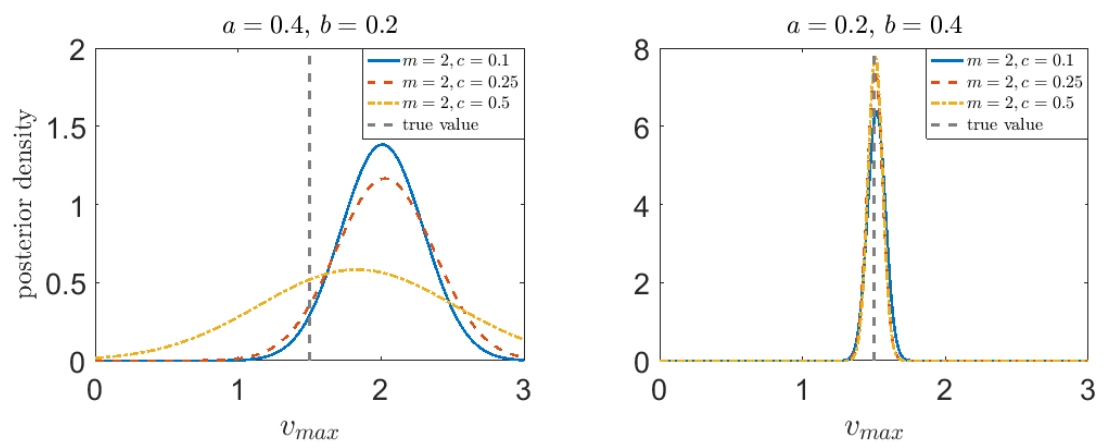

FIG. 6.8. Influence of prior variance for two different regimes using solutions of the steady state equation. Left: Outflux limited regime with $a=0.4, b=0.2$. Right: Influx limited regime with $a=0.2, b=0.4$.

which mimics the prior.

Influence of information and the proximity of the parameters $a$ and $b$. Next we discuss how the amount of information, that is, the number of trajectories, and the ratio of the parameters $a$ and $b$ influence the quality of the estimates. The ratio of $a$ and $b$ determines the location of the boundary layer and the density range experienced by the trajectories. For example, the pair $a=0.2, b=0.4$ gives us a steady state profile with values for $\rho_{s}$ varying from $[0.2,0.5]$, while $a=0.1, b=0.15$ gives values in $[0.075,0.65]$.

We will show that the number of trajectories $J$ plays a large influence when $a \approx b$, while this is not the case when these parameters differ by a "large" amount. In this set of experiments only, we use the same value of $\Sigma$ to generate the data and in the inference method employed: $\sigma_{1}=\sigma_{2}=\sigma_{0}=0.05$. Figures 6.9 and 6.10 illustrate the role of $J$ and of the relative size of $a$ and $b$. In each of the panels, we vary the total number of trajectories, that is, $J=5,10,15,20$, and study the effect in two influx limited regimes.

We observe that, especially in the regime when the ratio between $a$ and $b$ is farther from 1, the estimates become better as the number of trajectories increases, both in the estimate itself (mean of the posterior distribution) and in the variance of 

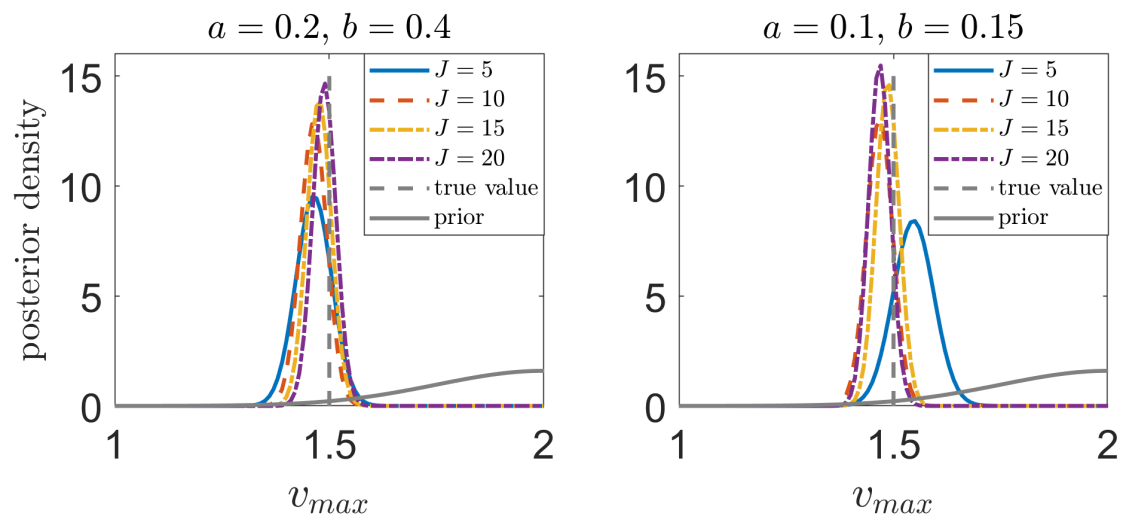

FIG. 6.9. Influence of the number of trajectories on the estimate for $v_{\max }$ for influx limited regimes and in the time dependent case. Left: $a=0.2, b=0.4$. Right: $a=0.1, b=0.15$.

the posterior distribution. A similar behavior is observed for the steady state case, which is presented in Figure 6.10 below.
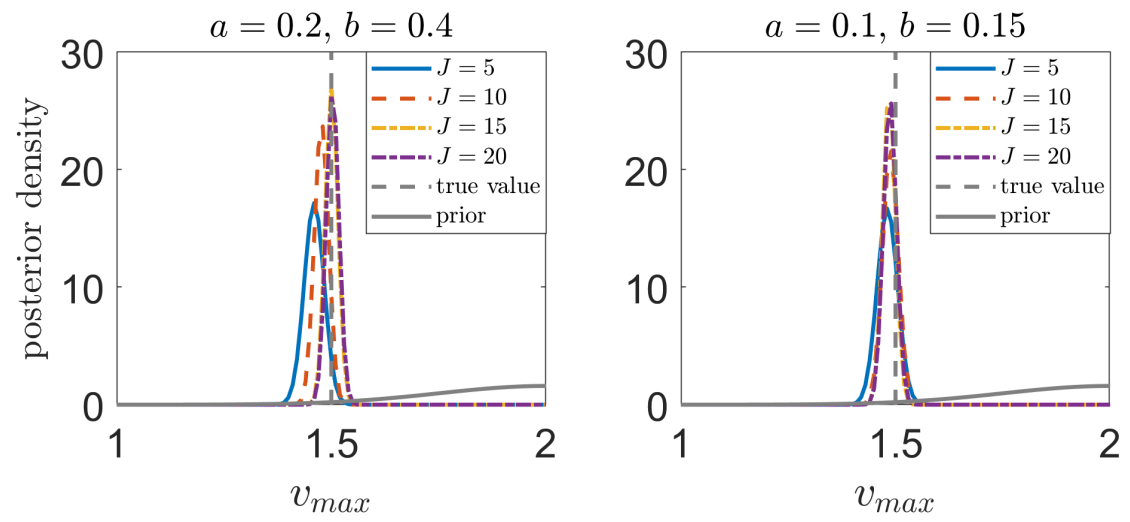

FIG. 6.10. Influence of the number of trajectories on the estimate for $v_{\max }$ for influx limited regimes, in the steady state regime. Left: $a=0.2, b=0.4$. Right: $a=0.1, b=0.15$.

Again, the estimate becomes closer to the true value, and with a smaller variance, as the number of trajectories increases. Furthermore, the regime where $a / b$ is farther from one has better estimates. This suggests that when planning experiments, these are preferable regimes to consider. We point out that Figures 6.9 and 6.10 are zoomed in so that the effect of the number of trajectories is clearly seen and, in particular, the prior is supported on a much larger length scale than that displayed, demonstrating that it has been forgotten.

Time dependent vs. steady state regimes. We have already pointed out that in a steady state situation, outflux limited regimes do not carry information about $v_{\max }$ due to the trajectories getting stuck. However, an important thing to notice is that in the influx limited and maximal current regimes the estimates for $v_{\max }$ are always accurate, and more importantly, less uncertain. This can be observed in, e.g., Figures 6.4 and 6.5 . 
6.3. Corridor with a bottleneck. We conclude with a more realistic example, in which pedestrians move through a corridor with a bottleneck. The corridor has length $L=3$ and a maximum width of $2 \ell=0.5$, which reduces to $2 w=0.1$ inside the bottleneck. The exit boundary is now split into a rigid wall (Neumann/reflecting boundary conditions) and a door (Robin/partially reflecting boundary conditions) which has width 0.3. We assume that diffusion in the vertical direction is smaller due to limited space inside the bottleneck. In particular we set $\sigma_{1}=0.05$ and $\sigma_{2}=0.03$. In this example the vector $e_{1}$ in (2.3) is replaced by the negative gradient of a potential $\phi$. This potential is computed from the eikonal equation with suitable boundary conditions; see [35]. The function $\phi$ corresponds to the shortest distance to the exit, and its negative gradient to the optimal trajectory to navigate towards a door and/or through a bottleneck; see Figure 6.11.

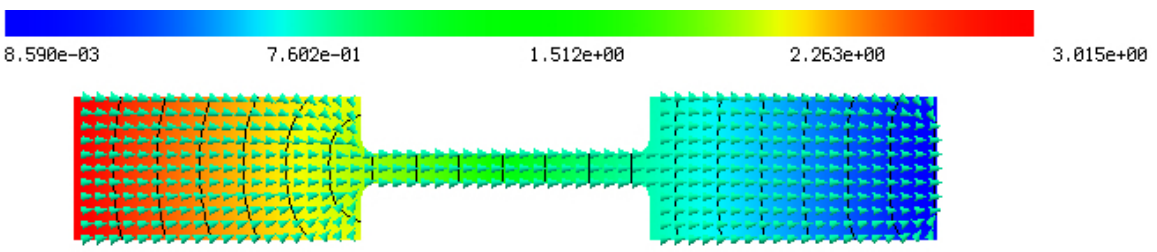

FIG. 6.11. Solution of the eikonal equation in the corridor with a bottleneck described in this section. The arrows depict the direction of the negative gradient, which is the direction in which individuals move.

We generate trajectories using a time step $\Delta t_{S D E}=10^{-3}$ and set the final time $T=1$. In the PDE solver the time step was set to $\Delta t_{P D E}=4 \times 10^{-3}$. The true value of $v_{\max }$ was, as before, $v_{\max }=1.5$, and we used two of the previous regimes: $a=0.2, b=0.4$ and $a=0.4, b=0.2$.
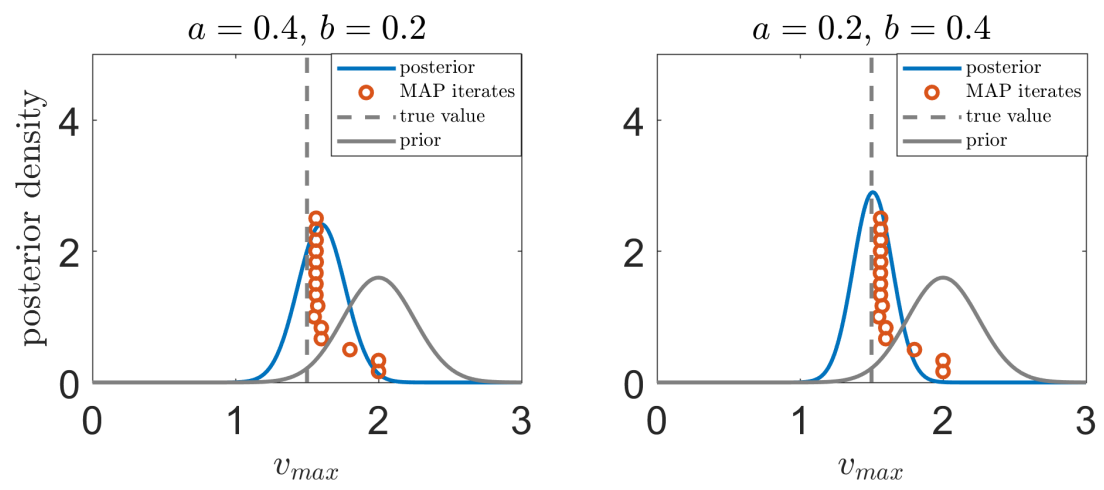

FIG. 6.12. Results of the parameter estimation methodology in a corridor with a bottleneck. Left: Outflux limited regime with $a=0.4, b=0.2$. Right: Influx limited regime with $a=0.2, b=0.4$.

In Figure 6.12 we present our numerical results. The figure depicts the true value of $v_{\max }$ (dashed gray line), prior distribution (solid gray line), posterior distribution (solid blue line), and the Nelder-Mead algorithm iterates (orange circles). We observe that both regimes produce very good estimates for $v_{\max }$. This demonstrates that the framework we have developed can be applied to more realistic setups; we intend to study the impact of parameters and geometry in more detail in future work. 
7. Conclusions/discussion. We have studied a macroscopic model for a unidirectional flow of pedestrians in a corridor. The evolution of the pedestrian density is given by a nonlinear Fokker-Planck equation, whose coefficients depend on the socalled fundamental diagram. We formulated and analyzed the problem of estimating two parameters of interest in the fundamental diagram using individual trajectories. We assume that these trajectories are realizations of a generalized McKean-Vlasov equation. This identification problem was solved using derivative-free methodologies. We have shown that the first parameter - the maximum pedestrian density - cannot be estimated from the model considered. The second characteristic quantity - the maximum speed - can be accurately learned from a variety of inflow and outflow conditions, in both time dependent and steady state settings. We have also seen that boundary conditions play an important role. We believe that the proposed framework may help one to understand their impact in estimation as well as experimental design.

We also believe that the developed framework provides the basis for future developments in applied mathematics as well as transportation research. In particular, the next steps include the identification of parameters in different forms of the fundamental diagram, or the application of nonparametric estimation techniques to learn its functional form. Furthermore, we want to use pedestrian trajectory data, which requires the framework to be generalized to noisy observations.

These future developments will contribute to the validation of the fundamental diagram adopted in many models in the transportation literature. It will also justify its use in certain parameter regimes for microscopic pedestrian models.

\section{Appendix A.}

A.1. Proof of Theorem 3.1 and Corollary A.2. Equation (2.5a) is a gradient flow with respect to the entropy functional

$$
E(\rho)=\int_{\Omega}(\rho \log \rho+(1-\rho) \log (1-\rho)-\rho V) d x
$$

with $V(x)=x_{1}$. The corresponding entropy variable $u=\frac{\delta E}{\delta \rho}=\log \rho-\log (1-\rho)-V$ allows us to write $(2.5 \mathrm{a})$ as

$$
\partial_{t} \rho=\operatorname{div}(m(\rho) \nabla u)
$$

with a nonlinear mobility $m(\rho)=\rho(1-\rho)$. This gradient flow structure provides the necessary a priori estimates to prove global in time existence of solutions.

Proof of Theorem 3.1. Let $N \in \mathbb{N}$, and consider a discretization of $(0, T]$ into subintervals $(0, T]=\cup_{k=1}^{N}((k-1) \tau, k \tau]$ with time steps $\tau=\frac{T}{N}$. We look for a weak solution $\rho: \Omega \times[0, T] \rightarrow \mathcal{S}$ of (2.5a) in the sense of (3.2). The proof is based on the implicit Euler discretization, which gives us a recursive sequence of elliptic problems. We consider its regularized version

$$
\frac{\rho_{k}-\rho_{k-1}}{\tau}=\operatorname{div}\left(m\left(\rho_{k}\right) \nabla u_{k}\right)+\tau \Delta u_{k} .
$$

Existence of at least one weak solution $\rho \in H^{1}(\Omega) \cap L^{\infty}(\Omega)$ with $0 \leq \rho \leq 1$ to (A.2) follows from Theorem 3.5 in [6] if assumptions (A1)-(A3) are satisfied. Note that the transformation from $\rho$ to the entropy variables $u$ is one to one and is given by

$$
\rho=\frac{e^{u+V}}{1+e^{u+V}} .
$$

Copyright ( by SIAM. Unauthorized reproduction of this article is prohibited. 
Therefore solutions $\rho$ automatically lie in the set $\mathcal{S}$. Furthermore the entropy density

$$
h(\rho)=\rho \log \rho-(1-\rho) \log (1-\rho)-\rho V
$$

is strictly convex for $\rho \in \mathcal{S}^{0}$ since $h^{\prime}(\rho)=\log \frac{\rho}{1-\rho}-V$ and $h^{\prime \prime}(\rho)=\frac{1}{\rho}+\frac{1}{1-\rho}$. Since $h$ is convex we have that $h\left(q_{1}\right)-h\left(q_{2}\right) \leq h^{\prime}\left(q_{1}\right)\left(q_{1}-q_{2}\right)$, and therefore for $q_{1}=\rho_{k}$ and $q_{2}=\rho_{k-1}$ that

$$
h\left(\rho_{k}\right)-h\left(\rho_{k-1}\right) \leq h^{\prime}\left(\rho_{k}\right)\left(\rho_{k}-\rho_{k-1}\right) .
$$

Entropy dissipation. We consider the weak formulation of (A.2) to obtain

$$
\begin{aligned}
\frac{1}{\tau} \int_{\Omega}\left(\rho-\rho_{k-1}\right) \varphi d x+\int_{\Omega} \nabla \varphi^{T} m\left(\rho_{k}\right) \nabla u_{k} d x-a & \int_{\Gamma_{\text {in }}}\left(1-\rho_{k}\right) \varphi d s \\
& +b \int_{\Gamma_{\text {out }}} \rho_{k} \varphi d s+\tau \int_{\Omega} \nabla \varphi \nabla u_{k}=0
\end{aligned}
$$

for $\varphi \in H^{1}(\Omega)$ and use that $\rho_{k}=h^{-1}\left(u_{k}\right)$. Since $u_{k}=h^{\prime}\left(\rho_{k}\right)$ we can rewrite (A.4) as

$$
\frac{1}{\tau} \int_{\Omega}\left(\rho_{k}-\rho_{k-1}\right) u_{k} d x \geq \frac{1}{\tau} \int_{\Omega}\left[h\left(\rho_{k}\right)-h\left(\rho_{k-1}\right)\right] d x .
$$

Using (A.5) and choosing test functions $\varphi=u_{k}$ gives

$$
\begin{aligned}
\int_{\Omega} h\left(\rho_{k}\right) d x+\tau & \int_{\Omega} \nabla u_{k}^{T} m\left(\rho_{k}\right) \nabla u_{k} d x-a \tau \int_{\Gamma_{\text {in }}}\left(1-\rho_{k}\right) u_{k} d s \\
& +b \tau \int_{\Gamma_{\text {out }}} \rho_{k} u_{k} d s+\tau^{2} \int_{\Omega} \nabla u_{k} \nabla u_{k} \leq \int_{\Omega} h\left(\rho_{k-1}\right) d x .
\end{aligned}
$$

Next we prove the following a priori estimate.

Lemma A.1. Let $\rho \in L^{2}(\Omega)$ and $\rho \in \mathcal{S}^{0}$ a.e. be such that $u=h^{\prime}(\rho) \in H^{1}(\Omega)$. Then there exist constants such that

$$
\int_{\Omega} \nabla u^{T} m(\rho) \nabla u-a \int_{\Gamma_{\text {in }}}(1-\rho) u d s+b \int_{\Gamma_{\text {out }}} \rho u d s \geq \int_{\Omega}|\nabla \rho|^{2} d x-C .
$$

Proof. For the first term we obtain

$$
\int_{\Omega} \nabla u^{T} m(\rho) \nabla u d x=\int_{\Omega} \rho(1-\rho)|\nabla u|^{2} d x .
$$

Since $\nabla u=\frac{\nabla \rho}{\rho(1-\rho)}-\nabla V$ we have

$$
\begin{aligned}
\int_{\Omega} \nabla u^{T} m(\rho) \nabla u d x & =\int_{\Omega} \frac{|\nabla \rho|^{2}}{\rho(1-\rho)} d x-2 \int_{\Omega} \nabla V \cdot \nabla \rho+\int_{\Omega} \rho(1-\rho)|\nabla V|^{2} d x \\
& \geq \int_{\Omega} \frac{|\nabla \rho|^{2}}{2 \rho(1-\rho)}-\rho(1-\rho)|\nabla V|^{2} d x \\
& \geq 2 \int_{\Omega}|\nabla \rho|^{2} d x-\frac{1}{4} \int_{\Omega}|\nabla V|^{2} d x .
\end{aligned}
$$

Copyright $@$ by SIAM. Unauthorized reproduction of this article is prohibited. 
The estimates follow from Cauchy's inequality and the fact that $\rho(1-\rho) \leq \frac{1}{4}$. The inflow boundary term can be rewritten as

$$
\begin{aligned}
-\int_{\Gamma_{i n}}(1-\rho) u d s & =-\int_{\Gamma_{i n}}(1-\rho)(\log \rho-\log (1-\rho)-V) d s \\
& =\int_{\Gamma_{i n}}\left[(1-\rho) \log \frac{1-\rho}{\rho}+2 \rho-1\right] d s+\int_{\Gamma_{i n}}[(1-\rho) V-2 \rho+1] d s .
\end{aligned}
$$

The first term on the right-hand side is a Kullback-Leibler distance and therefore nonnegative. Since $V \in H^{1}(\Omega)$ we know that its trace satisfies $\left.V\right|_{\partial \Omega} \in L^{2}(\partial \Omega)$, and since $\rho \in \mathcal{S}$ the second term is bounded. We can use a similar argument for the outflow term, which can be written as

$$
\begin{aligned}
-\int_{\Gamma_{\text {out }}} \rho u d s & =-\int_{\Gamma_{\text {out }}} \rho(\log \rho-\log (1-\rho)-V) d s \\
& =\int_{\Gamma_{\text {out }}}\left[\rho \log \frac{1-\rho}{\rho}+2 \rho-1\right] d s+\int_{\Gamma_{\text {out }}}[\rho V-2 \rho+1] d s .
\end{aligned}
$$

Again we have a nonnegative and a bounded term on the right-hand side.

The dissipation inequality and the recursion yields

$$
\int_{\Omega} h\left(\rho_{k}\right) d x+\tau \sum_{j=1}^{k} \int_{\Omega}\left|\nabla \rho_{k}\right|^{2} d x+\tau^{2} \sum_{k=1}^{n} R\left(u_{k}, u_{k}\right) \leq \int_{\Omega} h\left(\rho_{0}\right) d x+T C,
$$

where $R(u, \varphi)=\int_{\Omega} \nabla u \nabla \varphi d x$. This discrete entropy dissipation relation allows us to pass to the limit $\tau \rightarrow 0$.

The limit $\tau \rightarrow 0$. Let $\rho_{k}$ denote a sequence of solutions to (A.2). We define $\rho_{\tau}(x, t)=\rho_{k}(x)$ for $x \in \Omega$ and $t \in((k-1) \tau, k \tau]$. Then, if $\sigma_{\tau}$ denotes the shift operator, i.e., $\left(\sigma_{\tau} \rho_{\tau}\right)(x, t)=\rho_{\tau}(x, t-\tau)$ for $\tau \leq t \leq T$, then $\rho_{\tau}$ solves the following problem:

$$
\begin{aligned}
\int_{0}^{T} \int_{\Omega}\left(\frac{1}{\tau}\left(\rho_{\tau}-\sigma_{\tau} \rho_{\tau}\right) \varphi+\nabla \rho_{\tau} \nabla \Phi-\rho_{\tau}\left(1-\rho_{\tau}\right) \nabla \varphi\right) d x \\
-a \int_{0}^{T} \int_{\Gamma_{\text {in }}}\left(1-\rho_{\tau}\right) \varphi d s+b \int_{\Gamma_{\text {out }}} \rho_{\tau} \varphi d s=0
\end{aligned}
$$

for test functions $\varphi \in L^{2}\left(0, T ; H^{1}(\Omega)\right)$. Then the entropy dissipation inequality becomes

$$
\int_{\Omega} h\left(\rho_{\tau}(T)\right) d x+\int_{0}^{T} \int_{\Omega}\left|\nabla \rho_{\tau}\right|^{2} d x+\tau \int_{0}^{T} R\left(u_{k}, u_{k}\right) \leq \int_{\Omega} h\left(\rho_{0}\right) d x+T C,
$$

which gives the a priori estimate $\left\|\rho_{\tau}\right\|_{L^{2}\left(0, T ; H^{1}(\Omega)\right)} \leq K$. Using the a priori estimates, we use (A.7) to obtain

$$
\int_{0}^{T} \int_{\Omega}\left\langle\rho_{\tau}-\sigma_{\tau} \rho, \varphi\right\rangle d t \leq K\|\varphi\|_{L^{2}\left(0, T ; H^{1}(\Omega)\right)} .
$$

We know that $\rho_{\tau}$ is in $L^{2}\left(0, T ; H^{1}(\Omega)\right)$ and $\frac{1}{\tau}\left\langle\rho_{\tau}-\sigma_{\tau} \rho_{\tau}\right\rangle$ in $L^{2}\left(0, T ; H^{1}(\Omega)^{*}\right)$. Therefore we can use Aubin's lemma to conclude the existence of a subsequence, also denoted by $\rho_{\tau}$ such that for $\tau \rightarrow 0$

$$
\rho_{\tau} \rightarrow \rho \quad \text { strongly in } L^{2}\left(0, T ; L^{2}(\Omega)\right) .
$$


Finally we check that all terms in (A.7) converge to the right limit as $\tau \rightarrow 0$. Because of (A.9) we know that

$$
1-\rho_{\tau} \rightarrow 1-\rho \quad \text { strongly in } L^{2}\left(0, T, L^{2}(\Omega)\right) .
$$

Since $V \in H^{1}(\Omega)$ we can pass to the limit in the boundary terms as well. This concludes the existence proof.

Corollary A.2. Let all assumptions of Theorem 3.1 be satisfied. Then every weak solution $\rho \in L^{2}\left(0, T ; H^{1}(\Omega)\right)$ is also in $C(\bar{\Omega} \times(0, T))$.

Proof of Corollary A.2. The assertion follows from a bootstrap argument. Since $\rho \in L^{2}\left(0, T ; H^{1}(\Omega)\right)$ we can rewrite $(2.5 \mathrm{a})$ as

$$
\begin{aligned}
\partial_{t} \rho(x, t)-\operatorname{div}(\Sigma \nabla \rho) & =-\operatorname{div}(h) & & \text { in } \Omega \times(0, T), \\
\rho(x, t) & =g(x) & & \text { in } \Gamma \times(0, T),
\end{aligned}
$$

where $h=\rho F(\rho) \in L^{2}\left(0, T ; L^{2}(\Omega)\right)$ and $g \in L^{2}\left(0, T ; L^{2}(\Gamma)\right)$; then

$$
\|\rho\|_{L^{\infty}\left(0, T ; L^{2}(\Omega)\right)} \leq\|h\|_{L^{2}\left(0, T ; L^{2}(\Omega)\right)}+\|g\|_{L^{2}\left(0, T ; L^{2}(\Gamma)\right)} .
$$

Since $\Delta V=0$ we can rewrite $(2.5 \mathrm{a})$ as

$$
\begin{array}{rlrl}
\partial_{t} \rho-\operatorname{div}(\Sigma \nabla \rho)+v \cdot \nabla \rho & =0 & & \text { in } \Omega \times(0, T), \\
(\nabla \rho+v \rho) \cdot n=g & & \text { in } \Gamma \times(0, T), \\
\rho(x, 0) & =0 & \text { in } \Omega,
\end{array}
$$

with $g \in L^{2}\left(0, T ; L^{q}(\Gamma)\right)$. This equation has a unique solution $\rho \in L^{q}\left(0, T ; W^{2, q}(\Omega)\right) \cap$ $W^{1, q}\left(0, T ; L^{q}(\Omega)\right)$. From Sobolev embeddings we deduce that $\rho \in C^{0}(\bar{\Omega} \times(0, T))$.

Acknowledgments. SG thanks Imperial College London for the use of computer facilities. The authors are grateful to Grigorios Pavliotis for helpful discussions.

\section{REFERENCES}

[1] A. Aggarwal, R. M. Colombo, and P. Goatin, Nonlocal systems of conservation laws in several space dimensions, SIAM J. Numer. Anal., 53 (2015), pp. 963-983, https://doi.org/ $10.1137 / 140975255$.

[2] A. Apte, C. K. R. T. Jones, And A. M. Stuart, A Bayesian approach to Lagrangian data assimilation, Tellus A, 60 (2008), pp. 336-347.

[3] U. M. Ascher, S. J. RuUth, and B. T. R. Wetton, Implicit-explicit methods for timedependent partial differential equations, SIAM J. Numer. Anal., 32 (1995), pp. 797-823, https://doi.org/10.1137/0732037.

[4] N. W. F. Bode, M. Chraibi, And S. Holl, The emergence of macroscopic interactions between intersecting pedestrian streams, Transportation Research Part B: Methodological, 119 (2019), pp. 197-210.

[5] M. Boltes, A. Seyfried, B. Steffen, And A. Schadschneider, Automatic extraction of pedestrian trajectories from video recordings, in Pedestrian and Evacuation Dynamics 2008, Springer, 2010, pp. 43-54.

[6] M. Burger and J.-F. Pietschmann, Flow characteristics in a crowded transport model, Nonlinearity, 29 (2016), pp. 3528-3550.

[7] U. Chattaraj, A. Seyfried, and P. Chakroborty, Comparison of pedestrian fundamental diagram across cultures, Adv. Complex Syst., 12 (2009), pp. 393-405.

[8] R. M. Colombo And M. LÉCureux-Mercier, Nonlocal crowd dynamics models for several populations, Acta Math. Sci. Ser. B (Engl. Ed.), 32 (2012), pp. 177-196.

[9] A. Conn, K. Scheinberg, And L. N. Vicente, Introduction to Derivative-Free Optimization, SIAM, Philadelphia, 2009, https://doi.org/10.1137/1.9780898718768. 
[10] A. Corbetta, C.-M. Lee, R. Benzi, A. Muntean, and F. Toschi, Fluctuations around mean walking behaviors in diluted pedestrian flows, Phys. Rev. E, 95 (2017), 032316.

[11] S. L. Cotter, G. O. Roberts, A. M. Stuart, and D. White, MCMC methods for functions: Modifying old algorithms to make them faster, Statist. Sci., 28 (2013), pp. 424-446.

[12] E. Cristiani, B. Piccoli, And A. Tosin, Multiscale Modeling of Pedestrian Dynamics, MS\&A. Model. Simul. Appl. 12, Springer, Cham, 2014.

[13] D. A. Dawson, Critical dynamics and fluctuations for a mean-field model of cooperative behavior, J. Statist. Phys., 31 (1983), pp. 29-85.

[14] K. D. Elworthy, Stochastic Differential Equations on Manifolds, London Math. Soc. Lecture Note Ser. 70, Cambridge University Press, Cambridge, New York, 1982.

[15] R. Erban And S. J. Chapman, Reactive boundary conditions for stochastic simulations of reaction-diffusion processes, Phys. Biol., 4 (2007), pp. 16-28, http://stacks.iop.org/ $1478-3975 / 4 / \mathrm{i}=1 / \mathrm{a}=003$.

[16] S. M. Ermakov, V. V. Nekrutkin, and A. S. Sipin, Random Processes for Classical Equations of Mathematical Physics, Math. Appl. (Soviet Ser.) 34, Kluwer Academic Publishers, Dordrecht, 1989.

[17] J. Garnier, G. Papanicolaou, and T.-W. Yang, Mean Field Model for Collective Motion Bistability, preprint, https://arxiv.org/abs/1611.02194, 2016.

[18] J. Garnier, G. PapanicolaOU, And T.-W. Yang, Consensus convergence with stochastic effects, Vietnam J. Math., 45 (2017), pp. 51-75.

[19] J. GärTnER, On the McKean-Vlasov limit for interacting diffusions, Math. Nachr., 137 (1988), pp. $197-248$.

[20] K. Giesecke, G. Schwenkler, and J. Sirignano, Inference for Large Financial Systems, research paper, Boston University Questrom School of Business, Boston, 2018, https:// doi.org/10.2139/ssrn.3012751.

[21] P. Goatin and M. Mimault, A mixed system modeling two-directional pedestrian flows, Math. Biosci. Eng., 12 (2015), pp. 375-392.

[22] M. Hairer, A. M. Stuart, And J. Voss, Analysis of SPDEs arising in path sampling part II: The nonlinear case, Ann. Appl. Probab., 17 (2007), pp. 1657-1706.

[23] D. Helbing and P. Molnar, Social force model for pedestrian dynamics, Phys. Rev. E, 51 (1995), pp. 4282-4286.

[24] D. J. Higham, An algorithmic introduction to numerical simulation of stochastic differential equations, SIAM Rev., 43 (2001), pp. 525-546, https://doi.org/10.1137/ S0036144500378302.

[25] R. L. Hughes, The flow of human crowds, Annu. Rev. Fluid Mech., 35 (2003), pp. 169-182.

[26] S. M. IAcus, Simulation and Inference for Stochastic Differential Equations: With R Examples, Springer Science \& Business Media, 2008.

[27] J. P. Kaipio And E. Somersalo, Statistical and Computational Inverse Problems, Appl. Math. Sci. 160, Springer, 2005.

[28] Y. A. Kutoyants, Statistical Inference for Ergodic Diffusion Processes, Springer, 2004.

[29] J. C. Lagarias, J. A. Reeds, M. H. Wright, and P. E. Wright, Convergence properties of the Nelder-Mead simplex method in low dimensions, SIAM J. Optim., 9 (1998), pp. 112147, https://doi.org/10.1137/S1052623496303470.

[30] M. J. Lighthill and G. B. Whitham, On kinematic waves. II. A theory of traffic flow on long crowded roads, Proc. Roy. Soc. London. Ser. A, 229 (1955), pp. 317-345.

[31] J. A. Nelder and R. Mead, A simplex method for function minimization, Comput. J., 7 (1965), pp. 308-313.

[32] K. Oelschlager, A martingale approach to the law of large numbers for weakly interacting stochastic processes, Ann. Probab., 12 (1984), pp. 458-479.

[33] B. Øksendal, Stochastic Differential Equations: An Introduction with Applications, 6th ed., Springer-Verlag, Berlin, 2003.

[34] G. A. Pavliotis and A. M. Stuart, Parameter estimation for multiscale diffusions, J. Stat. Phys., 127 (2007), pp. 741-781.

[35] J. QIAN, Y. ZhANG, AND H. ZhaO, Fast sweeping methods for eikonal equations on triangular meshes, SIAM J. Numer. Anal., 45 (2007), pp. 83-107, https://doi.org/10.1137/050627083.

[36] B. L. S. Prakasa Rao, Statistical Inference for Diffusion Type Processes, Kendall's Lib. Statist. 8, Edward Arnold, London; Oxford University Press, New York, 1999.

[37] G. O. Roberts And O. Stramer, On inference for partially observed nonlinear diffusion models using the Metropolis-Hastings algorithm, Biometrika, 88 (2001), pp. 603-621.

[38] J. SchöBERL, Netgen/NGSolve, https://ngsolve.org/, 2010.

[39] A. Seyfried, B. Steffen, W. Klingsch, and M. Boltes, The fundamental diagram of pedestrian movement revisited, J. Stat. Mech. Theory Exp., 2005 (2005), P10002.

Copyright $@$ by SIAM. Unauthorized reproduction of this article is prohibited. 
[40] A. Singer, Z. Schuss, A. Osipov, and D. Holcman, Partially reflected diffusion, SIAM J. Appl. Math., 68 (2008), pp. 844-868, https://doi.org/10.1137/060663258.

[41] A. V. Skorokhod, Stochastic equations for diffusion processes in a bounded region, Theory Probab. Appl., 6 (1961), pp. 264-274.

[42] A. J. Wood, A totally asymmetric exclusion process with stochastically mediated entrance and exit, J. Phys. A, 42 (2009), 445002.

[43] J. Zhang, W. Klingsch, A. Schadschneider, And A. Seyfried, Ordering in bidirectional pedestrian flows and its influence on the fundamental diagram, J. Stat. Mech. Theory Exp., 2012 (2012), P02002.

Copyright (c) by SIAM. Unauthorized reproduction of this article is prohibited. 